\title{
Prevalence of Optimal Metabolic Health in American Adults: National Health and Nutrition Examination Survey 2009-2016
}

\author{
Joana Araújo, $\mathrm{PhD},{ }^{1}$ Jianwen $\mathrm{Cai}, \mathrm{PhD},{ }^{2}$ and June Stevens, $\mathrm{PhD}^{1,3}$
}

\begin{abstract}
Background: Several guidelines for cardiometabolic risk factor identification and management have been released in recent years, but there are no estimates of current prevalence of metabolic health among adults in the United States. We estimated the proportion of American adults with optimal cardiometabolic health, using different guidelines.

Methods: Data from the National Health and Nutrition Examination Survey 2009-2016 were analyzed $(n=8721)$. Using the most recent guidelines, metabolic health was defined as having optimal levels of waist circumference (WC $<102 / 88 \mathrm{~cm}$ for men/women), glucose (fasting glucose $<100 \mathrm{mg} / \mathrm{dL}$ and hemoglobin A1c $<5.7 \%$ ), blood pressure (systolic $<120$ and diastolic $<80 \mathrm{mmHg}$ ), triglycerides $(<150 \mathrm{mg} / \mathrm{dL}$ ), and high-density lipoprotein cholesterol $(\geq 40 / 50 \mathrm{mg} / \mathrm{dL}$ for men/women), and not taking any related medication.

Results: Changing from ATP III (Adult Treatment Panel III) guidelines to more recent cut points decreased the proportion of metabolically healthy Americans from 19.9\% (95\% confidence interval [CI]: 18.3-21.5) to $12.2 \%$ (95\% CI: 10.9-13.6). Dropping WC from the definition increased the percentage of adults with optimal metabolic health to $17.6 \%$. Characteristics associated with greater prevalence of metabolic health were female gender, youth, more education, never smoking, practicing vigorous physical activity, and low body mass index. Less than one-third of normal weight adults were metabolically healthy and the prevalence decreased to $8.0 \%$ and $0.5 \%$ in overweight and obese individuals, respectively.

Conclusions: Prevalence of metabolic health in American adults is alarmingly low, even in normal weight individuals. The large number of people not achieving optimal levels of risk factors, even in low-risk groups, has serious implications for public health.
\end{abstract}

Keywords: metabolic health, risk factors, prevalence

\section{Introduction}

$\mathrm{S}$ NCE 2001 a NUMBER of guidelines have been issued by American scientific societies and government agencies ${ }^{1-7}$ to provide evidence-based recommendations for cardiometabolic risk factor identification and management. Risk factor cut points indicate levels at which lifestyle and/or pharmaceutical intervention is advised, and provide de facto indicators to define metabolically healthy levels of traditional cardiometabolic risk factors. Currently there is no strong consensus on the combination of variables that define "metabolic health," and different subsets are used in the literature. ${ }^{8-10}$
We consider metabolic health to be related to, but not the same as, the absence of metabolic syndrome. The 2001 report from the National Cholesterol Education Program Adult Treatment Panel III (ATP III) ${ }^{1}$ helped to solidify the definition of metabolic syndrome as failing to meet optimal levels of three of five traditional cardiometabolic risk factors. The term metabolic syndrome was coined to point to clusters of risk factors - thus, the stipulation that multiple risk factors must be present. The term "metabolic health" is not aimed at clusters of risk factors, but at identification of a health status characterized by levels of metabolic indicators that are consistent with a high level of health and low risk of

Departments of ${ }^{1}$ Nutrition, ${ }^{2}$ Biostatistics, and ${ }^{3}$ Epidemiology, Gillings School of Global Public Health, University of North Carolina at Chapel Hill, Chapel Hill, North Carolina. 
impending cardiometabolic disease. The purpose of this research is to determine the number and proportion of American adults who have optimal levels of each of five traditional cardiometabolic risk factors in the absence of pharmaceutical treatment. We examine the impact of the use of cut points proposed by different agencies on these estimates, and show the distributions of metabolic health within demographic and lifestyle categories.

\section{Methods}

\section{Study population}

We used data from 2009-2016 National Health and $\mathrm{Nu}-$ trition Examination Survey (NHANES). ${ }^{11}$ NHANES is a nationally representative survey of the U.S. noninstitutionalized residents, selected by a complex multistage probability sampling design. The study protocol was approved by the institutional review board at the Centers for Disease Control and Prevention (Atlanta, GA), and written informed consent was obtained from all participants. The analyses included men and nonpregnant women aged $\geq 20$ years who had a fasting blood sample $(n=9447)$. Participants with missing data on components of the metabolic health definition, body mass index (BMI), physical activity, smoking, or educational level were excluded, and the final sample included 8721 participants.

\section{Data collection}

Data from NHANES participants were collected through questionnaires both at home and in the mobile examination centers (MECs), and examination data, including anthropometrics, were obtained in the MECs, using standardized procedures by trained research staff. Detailed information on data collection is described in NHANES protocols. ${ }^{12}$ In our analyses of demographic and lifestyle factors we used gender, age, education, race/ethnicity, moderate and vigorous recreational physical activity, smoking, and BMI. Age groups used in the analysis were 20-39, 40-59, and 60 years and over, and race/ethnicity was categorized as nonHispanic white, non-Hispanic black, Mexican American and other, according to the NHANES analytic guidelines. ${ }^{13}$ Education was grouped in three categories: less than high school, high school or equivalent, and at least some college. Self-reported data about any current practice of moderate and vigorous recreational physical activity was assessed using yes/no questions, which were then combined to create a three-option variable: none, only moderate, and at least some vigorous physical activity. Regarding smoking, never smokers were those participants who have not smoked at least 100 cigarettes in life, former smokers reported having smoked at least 100 cigarettes in life but do not smoke at all currently, and current smokers are those smoking currently, either everyday or some days. BMI used in the analysis was based on measured weight and height, and subjects were classified in four categories: underweight, $<18.5 \mathrm{~kg} / \mathrm{m}^{2}$; normal weight, $18.5-24.9 \mathrm{~kg} / \mathrm{m}^{2}$; overweight, $25.0-29.9 \mathrm{~kg} / \mathrm{m}^{2}$; and obese, $\geq 30.0 \mathrm{~kg} / \mathrm{m}^{2}$.

Regarding the cardiometabolic risk factors, waist circumference (WC) was assessed with a measuring tape at the uppermost lateral border of the right ilium to the nearest $0.1 \mathrm{~cm}$. After resting quietly in a seated position for $5 \mathrm{~min}$, three consecutive blood pressure readings were taken in the right arm. A fourth reading may have been taken, when a measurement was interrupted or incomplete. For the analysis, we used the average of the available readings, excluding the first reading. If only one blood pressure reading was available, that reading was used. Plasma glucose, glycated hemoglobin (HbA1c), triglycerides, and high-density lipoprotein cholesterol (HDL-C) were measured from fasting blood samples. Specific laboratory methods used to quantify each biochemical parameter are described in detail for each survey in NHANES files. ${ }^{14}$ Medication use for diabetes, blood pressure, and cholesterol was self-reported. Participants with missing data on self-reported medication were considered as not taking medication, if reported no previous medical diagnosis of the disease. The remaining missing values $(0.1 \%$ for blood pressure medication and $6.6 \%$ for cholesterol medication) were included in the denominator, when the prevalence of not taking medication was estimated.

\section{Definition of metabolic health}

Prevalence of metabolic health is the proportion of people who have optimal levels of all the risk factor variables without any medication. We first compared the prevalence of metabolic health using cut points from several scientific societies and government agencies. ${ }^{1-6}$ We then conducted a focused analysis of five risk factors, grouping variables (e.g., systolic and diastolic blood pressure [SBP and DBP]), and applying cut points using the most recent guidance: (i) $\mathrm{WC}<102 / 88 \mathrm{~cm}$ in men/women ${ }^{4}$; (ii) $\mathrm{SBP}<120 \mathrm{mmHg}$ and DBP $<80 \mathrm{mmHg}^{2,5}$; (iii) glucose $<100 \mathrm{mg} / \mathrm{dL}$ and $\mathrm{HbA} 1 \mathrm{c}$ $<5.7 \%$, ; (iv) triglycerides $<150 \mathrm{mg} / \mathrm{dL}^{4}$; (v) HDL-C $\geq 40 /$ $50 \mathrm{mg} / \mathrm{dL}$ in men/women. ${ }^{4}$ We also examined the prevalence of not taking medications for blood pressure, diabetes, and cholesterol.

\section{Statistical analysis}

Associations of optimal metabolic health with gender, age, education, race/ethnicity, physical activity, smoking, and BMI were examined in odds ratios. All analyses accounted for the complex survey design, using the fasting sampling weights and survey procedures (SURVEYFREQ and SURVEYLOGISTIC) in SAS 9.4 (Cary, NC), so estimates are representative of the noninstitutionalized civilian U.S. population. Although $<10 \%$ of subjects were excluded from our analysis due to missing data $(7.7 \%)$, we adjusted the NHANES fasting weights according to gender, race/ ethnicity, self-reported physical activity, and health insurance. ${ }^{13}$

Some guidelines ${ }^{5,7}$ recommend that levels of specific risk factors outside the normal range should be repeated on a separate day to avoid overestimation of risk factors prevalence. Since the NHANES study assessed each participant on only 1 day, we performed sensitivity analyses to examine the impact of correction for within-person day-to-day variability. The gender- and age-specific correction factors from Klungel et al. ${ }^{15}$ were applied to blood pressure, and the approximate average correction factor (0.8) was applied to glucose, HbA1c, HDL-C, and triglycerides. Since the ratio of the between-person variance to the within-person variance across days is generally smaller for blood pressure than for these other risk factors ${ }^{16-19}$ the sensitivity analysis was 
conservative, that is, likely identified more individuals as metabolically healthy than would a true correction.

\section{Results}

As expected, the proportion of metabolically healthy Americans changed depending on the cut points applied. Using the cut points from ATP III, ${ }^{1}$ the overall prevalence of metabolic health was $19.9 \%$ (95\% confidence interval: 18.3-21.5). It decreased to $16.0 \%(14.4-17.6)$ with the reduction of glucose threshold to $100 \mathrm{mg} / \mathrm{dL}^{4}$; to $14.8 \%$ (13.2-16.3) with the inclusion of HbA1c in the definition ${ }^{6}$; and finally to $12.2 \%(10.9-13.6)$ when the blood pressure cut points were lowered from $130 / 85$ to $120 / 80 \mathrm{mmHg}$ for SBP and DBP, respectively. ${ }^{2,5}$ The $12.2 \%$ prevalence corresponds to $\sim 27.3$ million American adults with optimal metabolic health.

Separate assessments of risk factors showed that $<50 \%$ of Americans had waist, glucose, and blood pressure levels in the optimal range, whereas almost three-quarters had triglycerides and HDL-C within optimal levels (Table 1). More than $90 \%$ reported not taking medication for diabetes, and around $70 \%$ did not take medication for blood pressure and cholesterol. In general, women had a higher prevalence of optimal cardiometabolic factors, although this was not true for WC or HDL-C: two risk factors with gender-specific cutoff. With the exception of lipids, the proportion of individuals with optimal risk factor levels tended to decrease with advancing age and higher BMI.

Using this set of the most recent and restrictive cutoffs (Fig. 1), the prevalence of metabolic health was higher in women than men, in those highly educated, who reported currently doing any recreational vigorous physical activity and never smokers; and it decreased markedly with aging. Less than one-third of normal weight adults were metabolically healthy, and the prevalence decreased with increasing BMI. The prevalence of metabolic health was higher among the underweight than in the normal weight; however, the percentage of Americans who are underweight is small and there were only 137 underweight adults in the NHANES unweighted sample. Exclusion of the WC criterion had a relatively large impact on the percentage of obese and overweight adults who were classified as metabolically healthy, increasing the percentages from $0.5 \%$ to $6.8 \%$ and from $8 \%$ to $15.0 \%$, respectively. Nevertheless, the prevalences were still much lower than seen in the normal weight and underweight groups. Removal of the WC criteria changed the overall estimate of optimal metabolic health from $12.2 \%$ to $17.6 \%$.

Table 2 shows the adjusted odds ratios computed to estimate the independent effects of each of the demographic and lifestyle factors shown in Fig. 1. All of the factors were independently associated with the odds of being metabolically healthy. The association between BMI and metabolic health was attenuated when WC was excluded from the metabolic health definition.

We combined the modifiable characteristics associated with higher or lower odds of metabolic health in a separate analysis to produce maximum contrast. This analysis showed that among normal weight adults, who had at least some college education, reported any vigorous physical activity regularly, and never smoked (population $n=5,660,191$ ), $46.4 \%$ enjoyed optimal metabolic health. In contrast, none
(0\%) had optimal metabolic health if they were obese, had less than a high school education, were not moderately or vigorously active, and were current smokers (population $n=2,777,388$ ).

Correction for short-term variability of the cardiometabolic risk factors hardly changed the estimated prevalence of metabolic health. After correction, prevalence of optimal levels of each factor changed by -0.2 percentage points for glucose metabolism (glucose and HbA1c) to 5.4 for HDL-C. However, the overall prevalence of metabolic health increased by only 1.1 percentage points (from $12.2 \%$ to $13.3 \%)$.

\section{Discussion}

Prevalence of optimal metabolic health is remarkably low among U.S. adults, with one in eight Americans achieving optimal levels of the five traditional cardiometabolic risk factors without medication. To our knowledge, no other study has provided similar estimates of metabolic health, although Muntner et al. $^{20}$ showed the impact of recent guidelines ${ }^{5}$ on the prevalence of hypertension. Other reports have described the prevalence of metabolic syndrome $e^{10,21}$ and combinations of risk factors and health behaviors ${ }^{8,22,23}$ using older data. In NHANES 1999-2004, 7.5\% of U.S. adults met criteria that included the absence of smoking, elevated BMI, elevated cholesterol, diabetes, and hypertension. ${ }^{8}$ Another study applied seven lifestyle and risk factors criteria suggested by the American Heart Association ${ }^{22}$ to national data from 2011 to 2012 and found that virtually $0 \%$ of U.S. adults met all the ideal levels (not smoking, having a healthy diet, sufficient physical activity, normal weight, and normal levels of total cholesterol, blood pressure, and glucose).$^{23}$ In this work, our focus is on physical manifestations of metabolic health, and we do not include health behaviors in the definition of metabolic health.

The low prevalence of optimal metabolic health was driven by the cut points used. These cut points were selected from prominent scientific societies and government agencies, and are therefore likely to be influential and evidence based. Nevertheless, they may not be entirely immune to advocacy for medication use, even though cutoffs used to identify optimal health are often stricter than those used to screen individuals eligible for pharmaceutical interventions. Recommendations of optimal levels of some risk factors have grown more restrictive for the past two decades as a result of evidence linking levels previously considered optimal to increased risk of cardiovascular disease. ${ }^{5,7,24,25}$ In this work, we did not consider adults taking medication for blood pressure, diabetes, or cholesterol metabolically healthy, even if cardiometabolic factors were within optimal levels. This option was consistent with the guidelines for the definition of metabolic syndrome. ${ }^{1,3,4}$ In addition, previous studies have shown that there is residual cardiovascular disease risk in patients treated for elevated blood pressure and cholesterol ${ }^{26-29}$ compared with adults with similar risk factor levels without pharmacologic treatment. Further, when the medication criteria were removed from the definition, prevalence of optimal metabolic health increased by only 1.2 percentage points.

We recognize that the evidence is weaker for elevated WC as a lone risk factor to define metabolic health compared with the other components; however, it is difficult to 


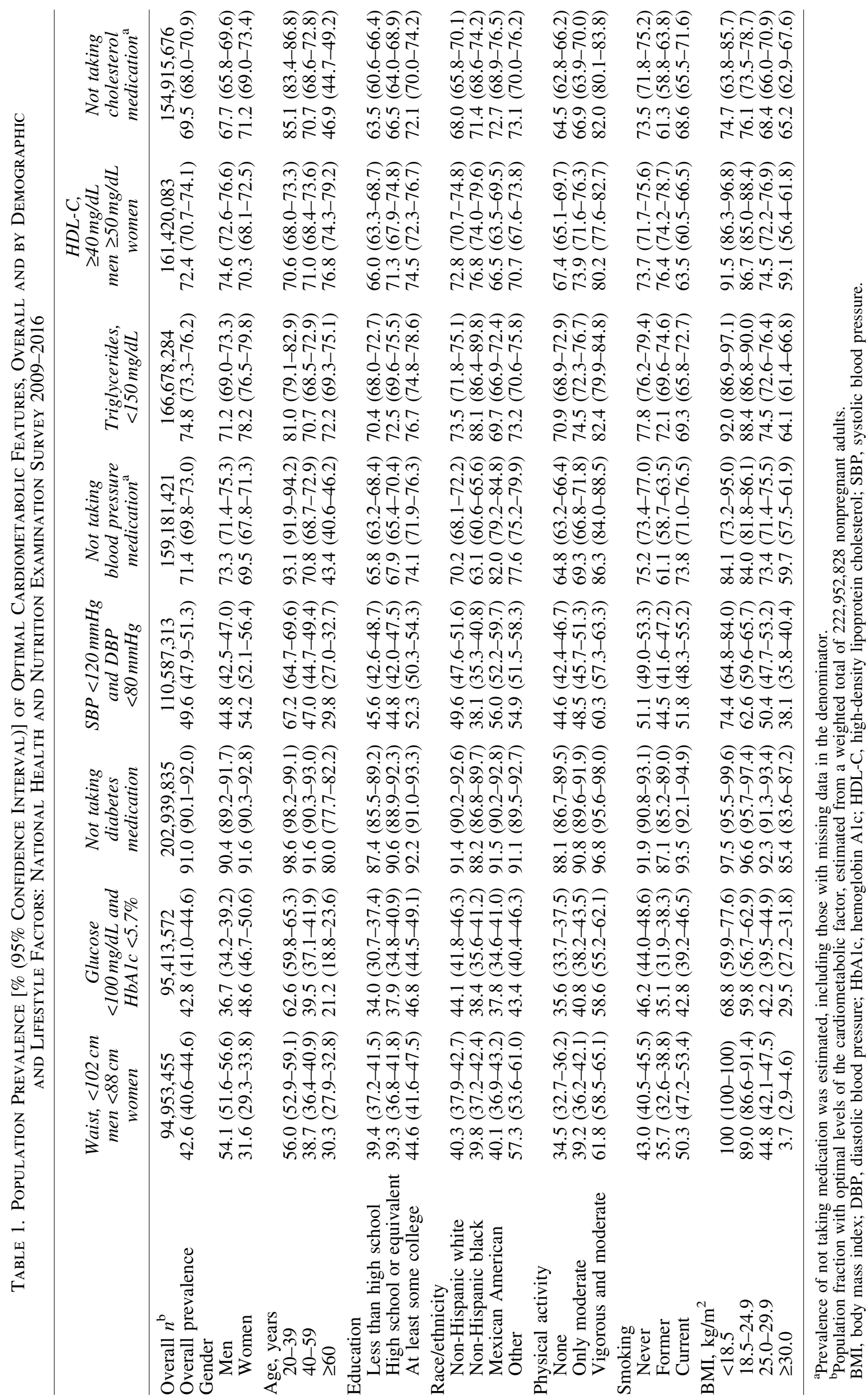




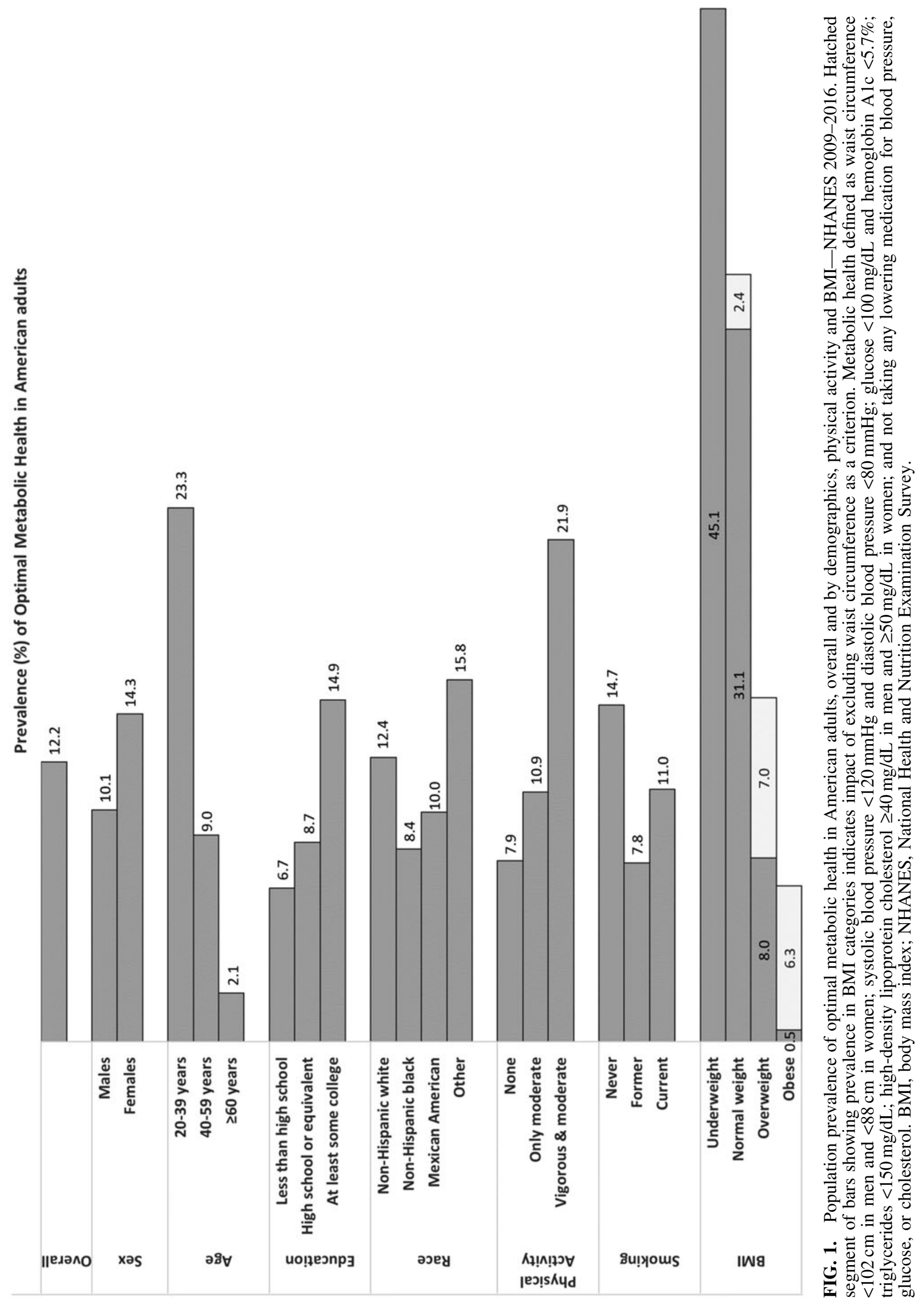


Table 2. Population Prevalence and Adjusted Odds Ratios of Optimal Cardiometabolic Health in American Adults, for Demographics, Physical Activity and Body Mass Index: National Health and Nutrition Examination Survey 2009-2016

\begin{tabular}{|c|c|c|}
\hline & $\%(95 \% C I)$ & $O R^{\mathrm{a}}(95 \% C I)$ \\
\hline \multicolumn{3}{|l|}{ Gender } \\
\hline Men & $10.1(8.5-11.6)$ & Ref. \\
\hline Women & $14.3(12.4-16.2)$ & $1.48(1.16-1.90)$ \\
\hline \multicolumn{3}{|l|}{ Age, years } \\
\hline $20-39$ & $23.3(20.7-26.0)$ & Ref. \\
\hline $40-59$ & $9.0(7.5-10.5)$ & $0.36(0.28-0.46)$ \\
\hline$\geq 60$ & $2.1(1.3-2.8)$ & $0.06(0.04-0.10)$ \\
\hline \multicolumn{3}{|l|}{ Education } \\
\hline $\begin{array}{l}\text { Less than high } \\
\text { school }\end{array}$ & $6.7(5.2-8.2)$ & Ref. \\
\hline $\begin{array}{l}\text { High school or } \\
\text { equivalent }\end{array}$ & $8.7(6.9-10.5)$ & $1.23(0.84-1.80)$ \\
\hline $\begin{array}{l}\text { At least some } \\
\text { college }\end{array}$ & $14.9(13.0-16.9)$ & $1.65(1.20-2.27)$ \\
\hline \multicolumn{3}{|l|}{ Race/ethnicity } \\
\hline Non-Hispanic white & $12.4(10.7-14.1)$ & Ref. \\
\hline Non-Hispanic black & $8.4(6.7-10.2)$ & $0.71(0.53-0.95)$ \\
\hline Mexican American & $10.0(8.2-11.7)$ & $0.85(0.63-1.15)$ \\
\hline Other & $15.8(13.2-18.3)$ & $0.76(0.60-0.97)$ \\
\hline \multicolumn{3}{|l|}{ Physical activity } \\
\hline None & $7.9(6.8-9.0)$ & Ref. \\
\hline Only moderate & $10.9(9.0-12.9)$ & $1.20(0.94-1.53)$ \\
\hline $\begin{array}{l}\text { Vigorous and } \\
\text { moderate }\end{array}$ & $21.9(19.0-24.9)$ & $1.54(1.22-1.95)$ \\
\hline \multicolumn{3}{|l|}{ Smoking } \\
\hline Never & $14.7(13.0-16.3)$ & Ref. \\
\hline Former & $7.8(6.1-9.5)$ & $0.77(0.56-1.05)$ \\
\hline Current & $11.0(8.8-13.1)$ & $0.60(0.46-0.79)$ \\
\hline \multicolumn{3}{|l|}{ BMI, $\mathrm{kg} / \mathrm{m}^{2}$} \\
\hline$<18.5$ & $45.1(35.0-55.3)$ & $1.89(1.24-2.86)$ \\
\hline $18.5-24.9$ & $31.1(28.0-34.2)$ & Ref. \\
\hline $25.0-29.9$ & $8.0(6.8-9.2)$ & $0.21(0.16-0.26)$ \\
\hline$\geq 30.0$ & $0.5(0.2-0.8)$ & $0.01(0.01-0.02)$ \\
\hline \multicolumn{3}{|l|}{ BMI, $\mathrm{kg} / \mathrm{m}^{2 \mathrm{~b}}$} \\
\hline$<18.5$ & $45.1(35.0-55.3)$ & $1.64(1.06-2.54)$ \\
\hline $18.5-24.9$ & $33.5(30.5-36.5)$ & Ref. \\
\hline $25.0-29.9$ & $15.0(13.2-16.8)$ & $0.40(0.33-0.49)$ \\
\hline$\geq 30.0$ & $6.8(5.5-8.1)$ & $0.15(0.12-0.19)$ \\
\hline
\end{tabular}

Statistically significant $(P<0.05)$ estimates are shown in bold. ${ }^{a}$ ORs were adjusted for all factors in the table.

${ }^{\mathrm{b}}$ Prevalence and odds ratio of optimal cardiometabolic health, excluding waist circumference as a criterion.

CI, confidence interval; OR, odds ratio.

justify labeling an adult with any one of the ATP III risk factors as having optimal metabolic health. Since WC is highly correlated with BMI, it is useful to examine associations of BMI with metabolic health with and without WC included in the definition. Dropping WC as a criterion increased the proportion of metabolic health by 5.4 percentage points overall, with greater impact in overweight and obese individuals. Even when WC was excluded from the definition, only one-third of the normal weight adults enjoyed optimal metabolic health. Given the roles of positive energy balance and excess adiposity in the accepted mechanistic genesis of several cardiometabolic risk factors, the meta- bolically unhealthy normal weight group is perplexing. Normal weight adults are at lower risk than heavier adults for development of cardiometabolic risk factors, ${ }^{30}$ but as illustrated in this study they are not immune, and it is known that metabolically unhealthy normal weight adults are at increased risk of all-cause mortality and cardiovascular events compared with the metabolically healthy normal weight. $^{31}$

The use of national data for this analysis is a strength, as was the recalibration of survey weights to better represent the American population. The lack of repeated measure of risk factors over different days was a study limitation, but our sensitivity analysis indicated that this deficit did not have a large impact on prevalence. Finally, cut points for continuous variables are at least somewhat arbitrary, and less strict cut points would result in a greater number of adults labeled as metabolically healthy.

\section{Conclusions}

The prevalence of metabolic health in American adults is very low, even in normal weight individuals. The large number of people not achieving optimal cardiometabolic levels of risk factors has serious implications for public health. More work is needed to understand the mechanisms of risk factor development, with attention given to normal weight as well as heavier adults. Most disturbing was the absence of optimal metabolic health in adults who had obesity, less than a high school education, were not physically active, and were current smokers. Currently available interventions to improve health-related behaviors have met with limited success, and stronger and more widely accessible strategies to promote healthier lifestyles are urgently warranted.

\section{Acknowledgment}

This study was not funded by any grant or financial support.

\section{Author Disclosure Statement}

No conflicting financial interests exist.

\section{References}

1. Expert Panel on Detection, Evaluation, and Treatment of High Blood Cholesterol in Adults. Executive summary of the Third Report of the National Cholesterol Education Program (NCEP) Expert Panel on Detection, Evaluation, and Treatment of High Blood Cholesterol in Adults (Adult Treatment Panel III). JAMA 2001;285:2486-2497.

2. Chobanian AV, Bakris GL, Black HR, et al. The Seventh Report of the Joint National Committee on Prevention, Detection, Evaluation, and Treatment of High Blood Pressure: The JNC 7 report. JAMA 2003;289:2560-2572.

3. Grundy SM, Cleeman JI, Daniels SR, et al. Diagnosis and management of the metabolic syndrome: An American Heart Association/National Heart, Lung, and Blood Institute Scientific Statement. Circulation 2005;112:27352752.

4. Alberti KG, Eckel RH, Grundy SM, et al. Harmonizing the metabolic syndrome: A joint interim statement of the International Diabetes Federation Task Force on Epidemiology and Prevention; National Heart, Lung, and Blood 
Institute; American Heart Association; World Heart Federation; International Atherosclerosis Society; and International Association for the Study of Obesity. Circulation 2009;120:1640-1645.

5. Whelton PK, Carey RM, Aronow WS, et al. 2017 ACC/ AHA/AAPA/ABC/ACPM/AGS/APhA/ASH/ASPC/NMA/ PCNA Guideline for the Prevention, Detection, Evaluation, and Management of High Blood Pressure in Adults: A report of the American College of Cardiology/American Heart Association Task Force on Clinical Practice Guidelines. J Am Coll Cardiol 2018;71:e127-e248.

6. American Diabetes Association. Diagnosis and classification of diabetes mellitus. Diabetes Care 2010;33(Suppl 1): S62-S69.

7. American Diabetes Association. 2. Classification and Diagnosis of Diabetes: Standards of Medical Care in Diabetes-2018. Diabetes Care 2018;41(Suppl 1):S13-S27.

8. Ford ES, Li C, Zhao G, et al. Trends in the prevalence of low risk factor burden for cardiovascular disease among United States adults. Circulation 2009;120:1181-1188.

9. Maclagan LC, Tu JV. Using the concept of ideal cardiovascular health to measure population health: A review. Curr Opin Cardiol 2015;30:518-524.

10. Ramphal L, Zhang J, Suzuki S. Ethnic disparities in the prevalence of the metabolic syndrome in American adults: Data from the Examination of National Health and Nutrition Examination Survey 1999-2010. Proc (Bayl Univ Med Cent) 2014;27:92-95.

11. Centers for Disease Control and Prevention (CDC). National Center for Health Statistics (NCHS). National Health and Nutrition Examination Survey. Accessed at https:// www.cdc.gov/nchs/nhanes/index.htm on January 11, 2018.

12. Centers for Disease Control and Prevention (CDC). National Center for Health Statistics (NCHS). National Health and Nutrition Examination Survey-Questionnaires, Datasets, and Related Documentation. Accessed at https://wwwn.cdc .gov/nchs/nhanes/Default.aspx on April 30, 2018.

13. Johnson CL, Paulose-Ram R, Ogden CL, et al. National health and nutrition examination survey: Analytic guidelines, 1999-2010. Vital Health Stat 2 2013:1-24.

14. Centers for Disease Control and Prevention (CDC). National Center for Health Statistics (NCHS). National Health and Nutrition Examination Survey-NHANES Laboratory Data. Accessed at https://wwwn.cdc.gov/nchs/nhanes/ search/datapage.aspx ?Component=Laboratory on May 7, 2018.

15. Klungel $\mathrm{OH}$, de Boer $\mathrm{A}$, Paes $\mathrm{AH}$, et al. Influence of correction for within-person variability in blood pressure on the prevalence, awareness, treatment, and control of hypertension. Am J Hypertens 2000;13(Pt 1):88-91.

16. Chambless LE, McMahon RP, Brown SA, et al. Short-term intraindividual variability in lipoprotein measurements: The Atherosclerosis Risk in Communities (ARIC) Study. Am J Epidemiol 1992;136:1069-1081.

17. Poon AK, Meyer ML, Reaven G, et al. Short-term repeatability of insulin resistance indexes in older adults: The atherosclerosis risk in communities study. J Clin Endocrinol Metab 2018;103:2175-2181.

18. Muntner P, Shimbo D, Tonelli M, et al. The relationship between visit-to-visit variability in systolic blood pressure and all-cause mortality in the general population: Findings from NHANES III, 1988 to 1994. Hypertension 2011;57: $160-166$.
19. Selvin E, Crainiceanu CM, Brancati FL, et al. Short-term variability in measures of glycemia and implications for the classification of diabetes. Arch Intern Med 2007;167:15451551.

20. Muntner P, Carey RM, Gidding S, et al. Potential US population impact of the 2017 ACC/AHA High Blood Pressure Guideline. Circulation 2018;137:109-118.

21. Ford ES, Li C, Zhao G. Prevalence and correlates of metabolic syndrome based on a harmonious definition among adults in the US. J Diabetes 2010;2:180-193.

22. Lloyd-Jones DM, Hong Y, Labarthe D, et al. Defining and setting national goals for cardiovascular health promotion and disease reduction: The American Heart Association's strategic Impact Goal through 2020 and beyond. Circulation 2010;121:586-613.

23. Mozaffarian D, Benjamin EJ, Go AS, et al. Heart disease and stroke statistics-2016 update: A report From the American Heart Association. Circulation 2016;133:e38e360.

24. Huang Y, Su L, Cai X, et al. Association of all-cause and cardiovascular mortality with prehypertension: A metaanalysis. Am Heart J 2014;167:160-168 e161.

25. Selvin E, Steffes MW, Zhu H, et al. Glycated hemoglobin, diabetes, and cardiovascular risk in nondiabetic adults. $N$ Engl J Med 2010;362:800-811.

26. Liu K, Colangelo LA, Daviglus ML, et al. Can antihypertensive treatment restore the risk of cardiovascular disease to ideal levels?: The Coronary Artery Risk Development in Young Adults (CARDIA) study and the Multi-Ethnic Study of Atherosclerosis (MESA). J Am Heart Assoc 2015;4: e002275.

27. Lieb W, Enserro DM, Sullivan LM, et al. Residual cardiovascular risk in individuals on blood pressure-lowering treatment. J Am Heart Assoc 2015;4:e002155.

28. Sampson UK, Fazio S, Linton MF. Residual cardiovascular risk despite optimal LDL cholesterol reduction with statins: The evidence, etiology, and therapeutic challenges. Curr Atheroscler Rep 2012;14:1-10.

29. Wong ND, Zhao Y, Quek RGW, et al. Residual atherosclerotic cardiovascular disease risk in statin-treated adults: The multi-ethnic study of atherosclerosis. J Clin Lipidol 2017;11:1223-1233.

30. Bradshaw PT, Reynolds KR, Wagenknecht LE, et al. Incidence of components of metabolic syndrome in the metabolically healthy obese over 9 years follow-up: The atherosclerosis risk in communities study. Int $J$ Obes (Lond) 2018;42:295-301.

31. Stefan N, Schick F, Haring HU. Causes, characteristics, and consequences of metabolically unhealthy normal weight in humans. Cell Metab 2017;26:292-300.

Address correspondence to: June Stevens, PhD Department of Nutrition Gillings School of Global Public Health University of North Carolina at Chapel Hill 2207 McGavran-Greenberg Hall CB \#7461

Chapel Hill, NC 27599

E-mail: june_stevens@unc.edu 